Supplemental Information

A Low-cost Beam Scanning Second Harmonic Generation Microscope and Application for Agrochemical Development and Testing

Benjamin A. Grubbs, Nicholas P. Etter, Wesley E. Slaughter, Alexander M. Pittsford, Connor R. Smith, and Paul D. Schmitt*

Department of Chemistry, Wabash College, Crawfordsville, IN 47933, United States.

*Corresponding author: schmittp@wabash.edu ORCID: 0000-0002-1460-5559

Data acquisition: synchronous digitization

The timing output of the fiber laser is coupled to an amplified photodiode (Thorlabs PDS10CF). This pulse train is sent to the PCle digital oscilloscope card (AlazarTech, model 9440) to serve as the master clock for data acquisition. As the $60 \mathrm{MHz}$ laser repetition rate of the laser source contains frequency contributions too high for the inherent bandwidth of the digital oscilloscope card (125 MHz), an inline 50 $\mathrm{MHz}$ low-pass filter was placed between the clock photodiode and the card to attenuate the higher frequency components of the signal (the frequency-space roll-off of this particular filter still allows a more than satisfactory portion of the $60 \mathrm{MHz}$ component to reach the card for clocking). The scanning mirrors are driven by a programmable digital-to-analog converter (DAC), which also provides a line-byline trigger signal for data acquisition (NI-9263 USB). This trigger signal is synchronous with the fast-axis mirror, such that each line represents an acquisition of 12,800 data points (50 laser pulses/ pixel across 256 pixels) synchronous with the firing of the laser (cable lengths were adjusted to ensure that the arrival of voltage transients from the PMT/ laser transmittance photodiode are in-phase with the laser clock signal). Each line of the image is stored in the on-board memory of the PCle digital oscilloscope care before being processed and reshaped into an image. Toward this end, either photon counting (based on a single threshold value of the digitized voltage) or signal averaging (storing the raw voltage value) can be performed for the PMT (SHG) image. Custom Matlab programs control the programming of the ADC, data acquisition, and image processing. Image analysis was performed using the automated particle-analysis packages available in NIH ImageJ. ${ }^{27}$

\title{
Platform validation:
}

It was found that the given objective lens and mirror displacement yielded a FOV size of $602 \times 602$ $\mu \mathrm{m}$ ( 256 x 256 pixels), assessed via laser bright field imaging of an NBS 1952 Resolution Target. A photograph of the microscope is given in Figure S1A showing (among other components), the scan head and post-scan head optical path, the PMT, the sample stage, and photodiode. As the photomultiplier tube used in acquisition of SHG images provides little inherent spectroscopic information (beyond the work function of the photo cathode), validation that the detector was imaging SHG and not another linear or nonlinear optical process (e.g., fluorescence or two-photon excited fluorescence) was also performed. While these alternative phenomena are unlikely to be imaged in this case $(1560 \mathrm{~nm}$ incident light is not likely to give rise to single or two-photon fluorescence due to insufficient energy for electronic excitation, in addition to the presence of a $785 \mathrm{~nm}$ bandpass filter and dichroic mirror prior to the PMT aperture), it is still prudent to provide thorough validation. Figure S1B shows the observed 
SHG intensity (average photon counts/ pixel) as a function of incident laser power. Error bars represent $\pm 1 \sigma$ if the experimental average is taken as the Poisson mean. The quadratic relationship observed is consistent with a second order nonlinear optical process such as SHG.

Preparation of the model extended-release wettable powder:

The extended release solids themselves were prepared via rotary evaporation of an active ingredient (griseofulvin) co-dissolved with a biodegradable polymeric adjuvant (HPMCAS) in methanol. (HPMCAS was verified to be SHG inactive via imaging of the raw powder in the solid state). The formulation was $50 \%$ active ingredient $(\mathrm{w} / \mathrm{w})$ dissolved in the minimum volume of methanol. Following rotary evaporation, the isolated solid was removed from the flask and ground extensively by hand in a mortar and pestle to reduce the particle size prior to suspension and application. The entire recovered mass ( $0.185 \mathrm{~g}$ ) was suspended in $13 \mathrm{~mL}$ DI water.

\section{Controls and blanks:}

First, blank leaves (not treated with any solvents, actives, or formulations) from each species (maize, glycine max, T. aestivum) were removed from the plant, affixed to a glass slide, and imaged across (a minimum of 10) randomly chosen fields of view. Example images are shown in the supporting information (Figure S2). Imaging at typical incident powers (20 $\mathrm{mW}$ at the back of the objective) did not reveal any discernable areas of SHG activity above the noise floor of the measurement in both wheat and corn leaves (background counts are statistically indistinguishable from images taken without a sample of any kind present, at 95\% confidence). An example image is shown in Figure S2A. In approximately $10 \%$ of cases, small areas of SHG activity were observed on soy bean leaves (Figure S2B). The source(s) of these signals are presently unknown, though are tentatively assumed to be arising from natural structures on the leaves (as opposed to a contaminant material. Visual inspection of soybean leaves does reveal the presence of small hair-like structures (trichomes) normal to the leaf surface, and the possibility that these single pixel signals come from the cross-sectional imaging of these structures is among the possibilities. When present, these single pixel signals were observed without any signal averaging at typical incident laser powers ( $20 \mathrm{~mW})$. Given their consistent size in the $\mathrm{x} / \mathrm{y}$ plane (typically 1 pixel, not observed to exceed 3 pixels), subsequent experiments on soy bean leaves in which automated particle identification or analysis was performed were set to require a minimum particle size of 4 pixels (providing a small buffer). While possibly excluding similarly-sized active ingredient crystals from the analyses, this strategy minimizes the likelihood that any of these naturally occurring signals from soy bean leaves are mistakenly attributed to an analyte of interest. A diverse series of additional controls to ensure observed SHG activity is arising from agrochemical active ingredient (vs. natural plant structures) were also performed and are described in detail in the Supporting Information.

A diverse series of negative controls was also performed. As leaves visibly dried out after removal from the plant, untreated leaves (no active ingredient present) were affixed to glass slides and imaged daily for up to two weeks. No SHG signal was observed, indicating that the drying process did not alter the leaf structure in a way that exposed or created SHG-active structures. Additionally, leaves of each species were adhered to glass slides, and appropriate volumes of each blank solvent or solvent mixture used in subsequent experiments were deposited onto the leaf and allowed to dry. Both of these controls (drying, application of carrier solvents) were also performed on leaves from each species harvested at different times in the plant's development. This collection of controls did not generate any SHG signal dissimilar from the phenomena described in the preceding paragraph. These combined results are 
consistent with natural plant structures not giving rise to observable SHG signal under the given imaging conditions in the vast majority of cases. Accordingly, any SHG signal arising from experiments in the presence of an SHG active small molecule (activity of the small molecule is confirmed beforehand via imaging the pure crystalline powder) is thus attributed to that crystalline small molecule.
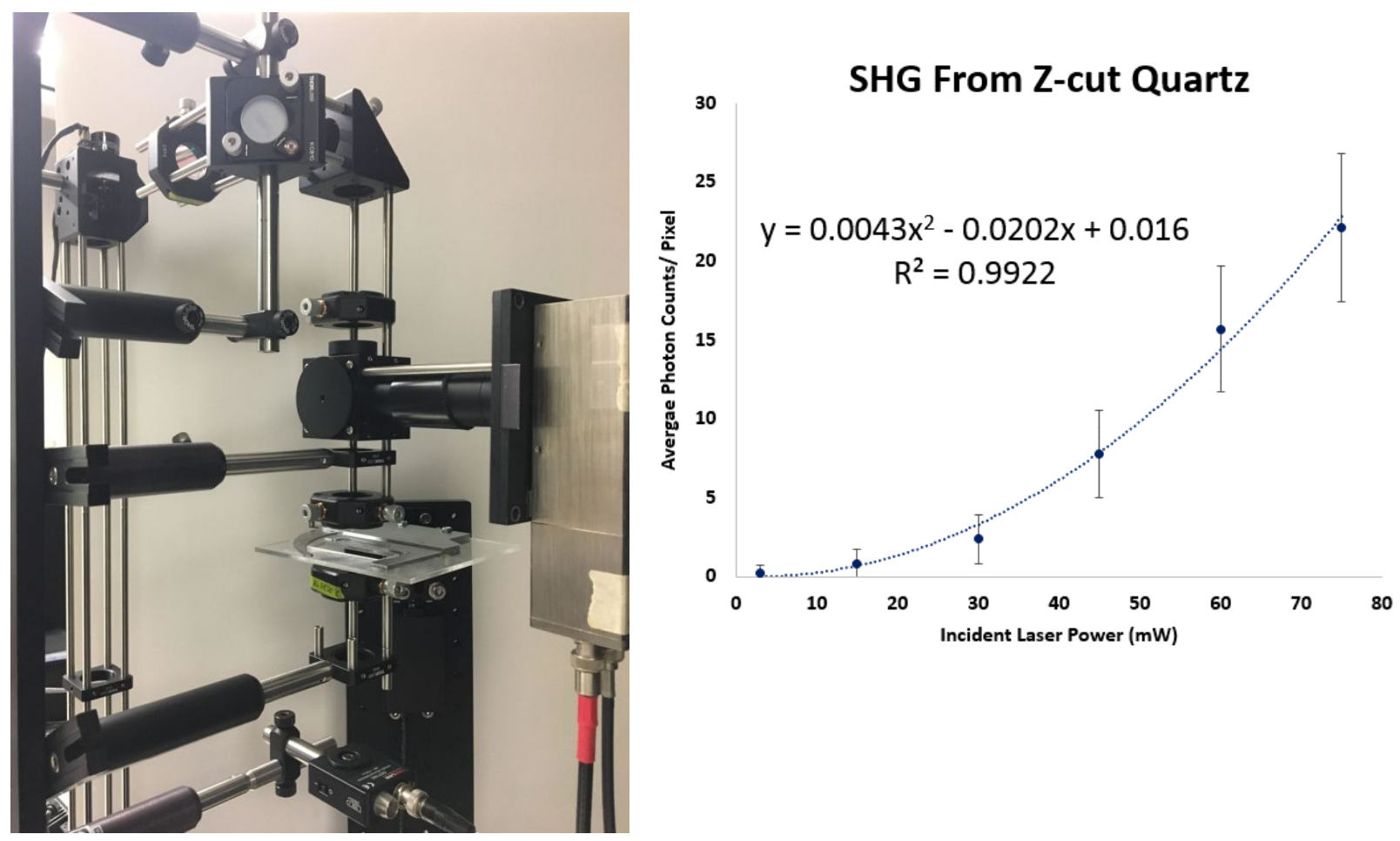

Figure S1: Photograph of the SHG microscope (left) and observed SHG signal as a function of incident laser power for z-cut quartz (right). The quadratic relationship is expected for SHG (a second order nonlinear optical process) and validated that the optical filters in front of the PMT enabled imaging of SHG. The error bars drawn on the plot indicate $\pm 1 \sigma$ if the measured value is taken as the Poisson mean. 


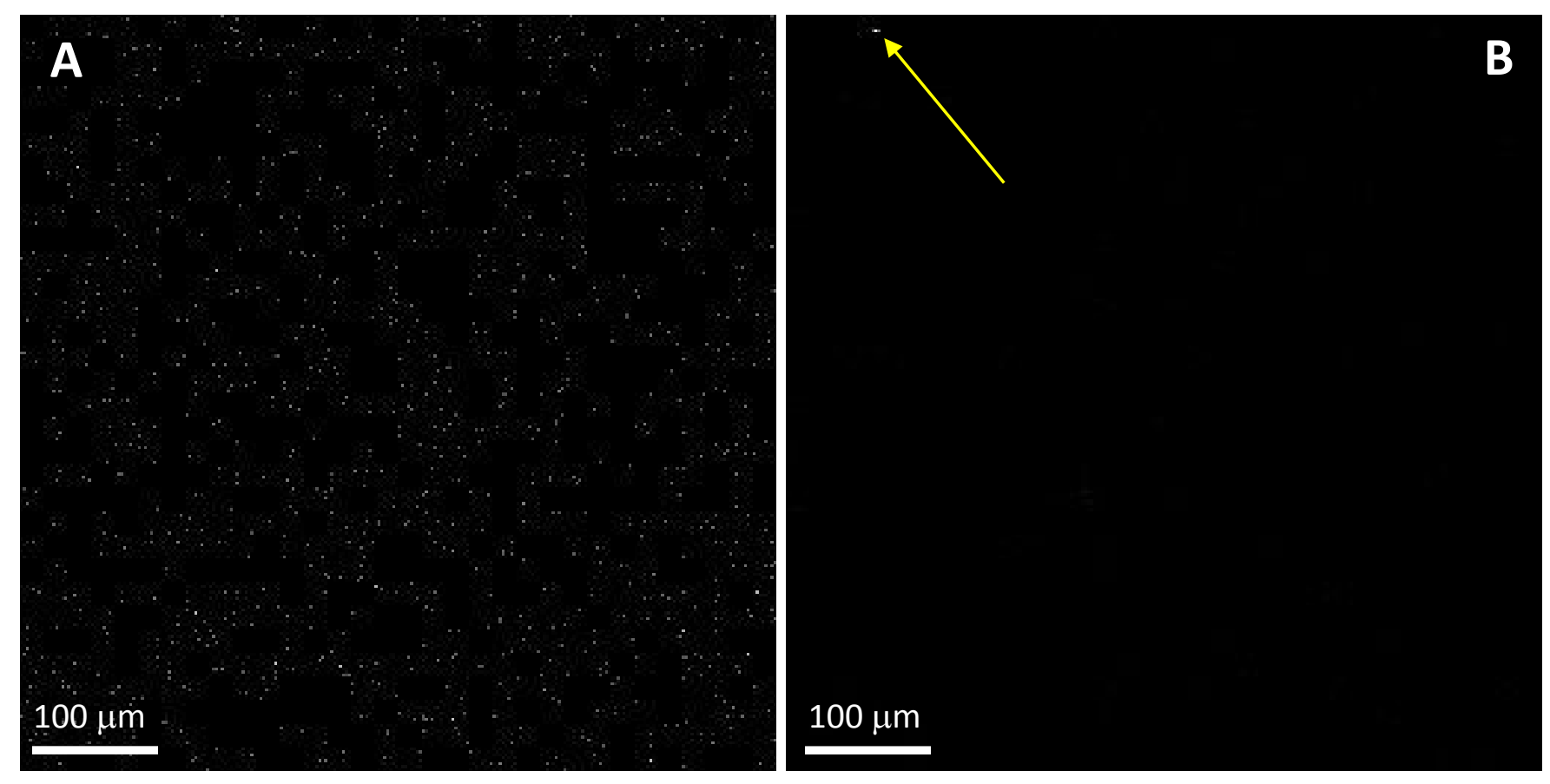

Figure S2: A: Example SHG image of an untreated corn leaf. The noise floor of the measurement is visible, and is statistically indistinguishable from a blank glass slide. B: small (3 pixel) area of SHG activity seen from an untreated soy leaf. The indicated area (the yellow arrow only serves to guide the eye) of SHG activity shows a maximum of 39 photon counts, well above the mean number of counts across the rest of the image ( 0.01). NB: under the given instrument settings, a maximum of 50 photon counts/ pixel is quantifiable given the singlethreshold photon counting algorithm (50 laser pulses/ pixel). 

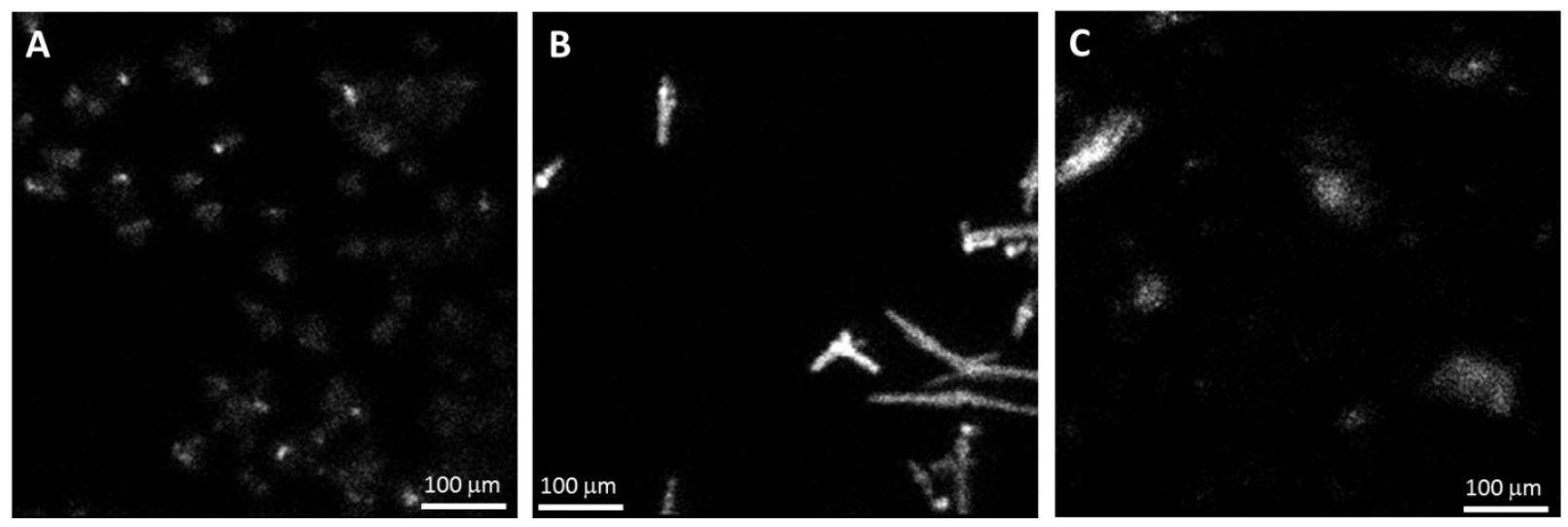

Figure S3: Representative SHG images of crystalline griseofulvin on soy, corn, and wheatgrass leaves ( $A, B$, and $C$, respectively) following solution-phase deposition. Changes in crystallization behavior are observed, with the active ingredient often (but not always) adopting a needle-like crystalline habit on corn leaves, but not on leaves of the other species. Characterization of the crystalline structures is 


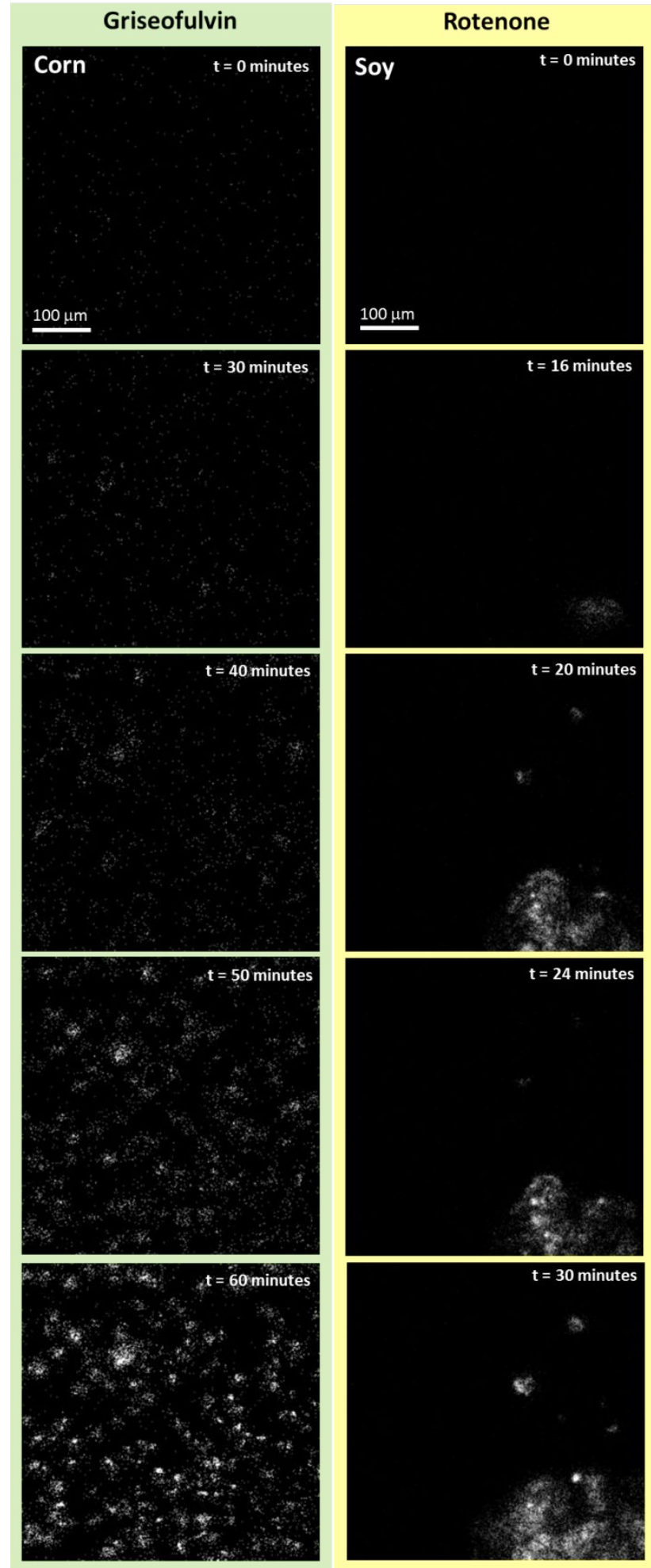

Figure S4: Additional still frames of real-time crystallization of griseofulvin on corn leaves (left column, green) and rotenone on soy bean leaves (right column, yellow). Full movies are also available. 

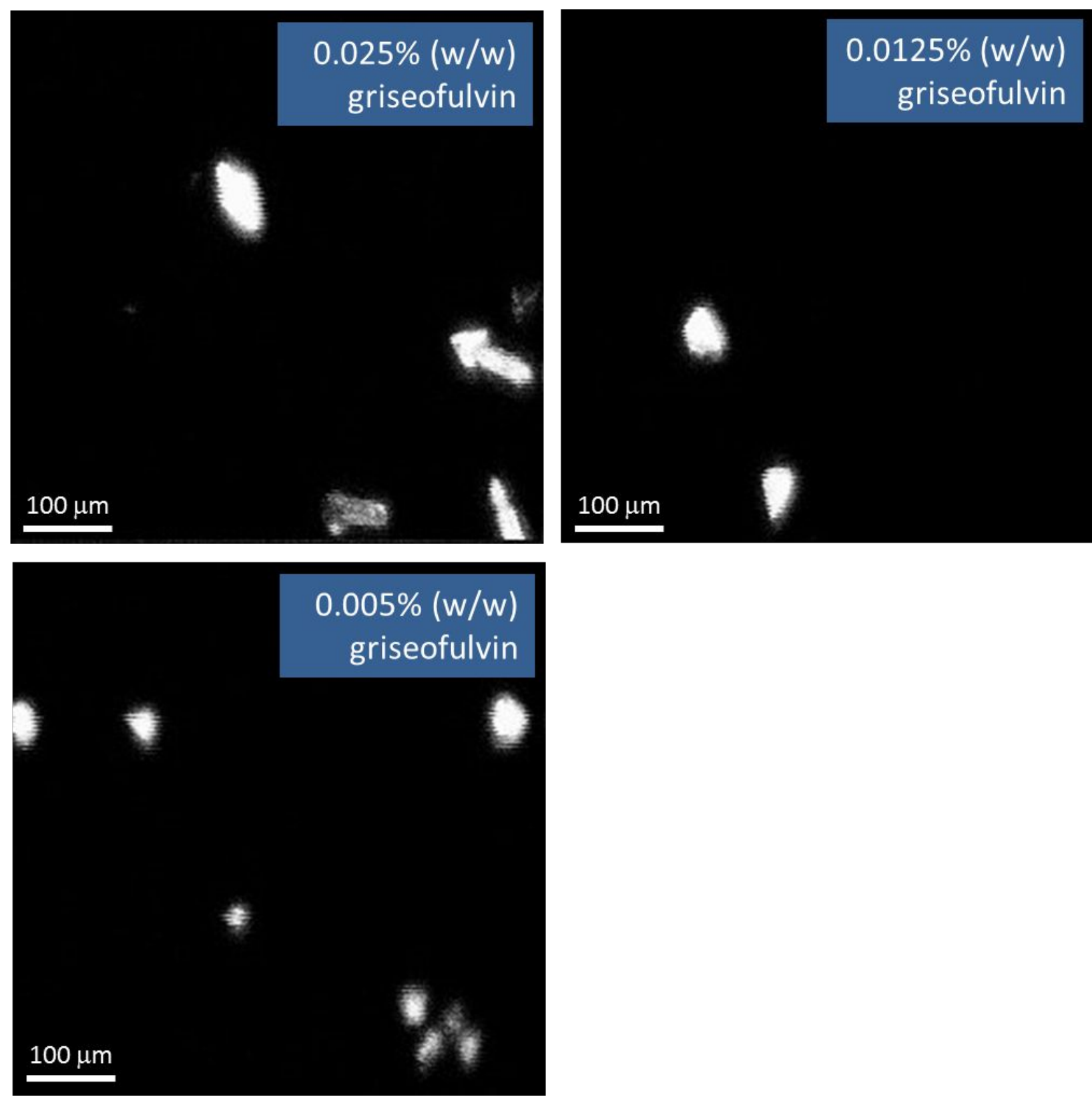

Figure S5: Representative SHG images of various dilutions of the fungicide griseofulvin following solution-phase deposition and drying onto soy leaves. The chosen concentrations are at or below common commercial active ingredient concentrations, highlighting i) crystallization occurring even at these lower concentrations and ii) the ability of SHG to image that crystallization. Unlike the insecticide rotenone (see Figure 5), these same solutions of griseofulvin showed crystallization when deposited onto glass slides (see Supporting Information, Figure S3). 


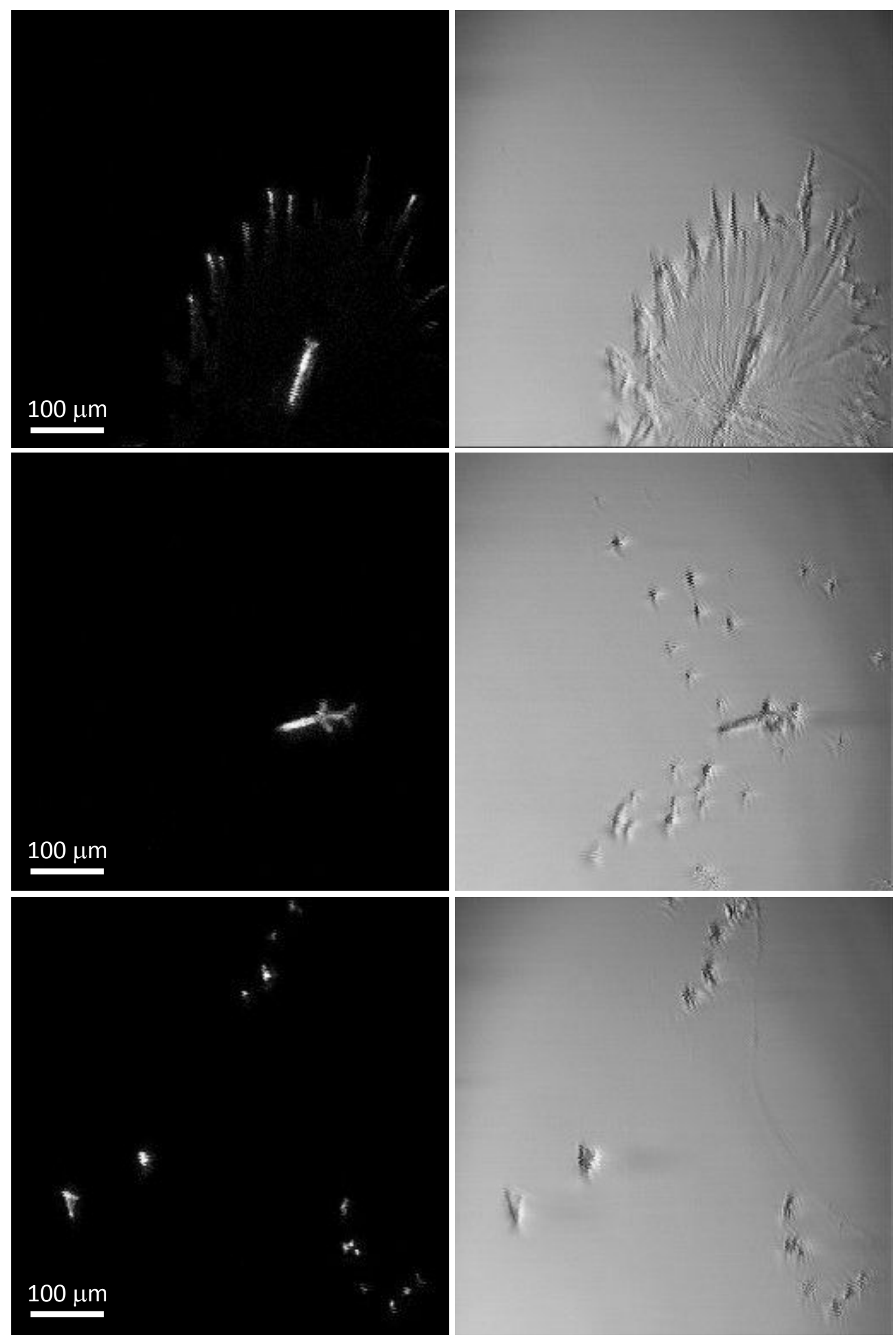

Figure S6: SHG images (left column) and corresponding laser bright field images (right column) of $0.025 \%$, $0.0125 \%$, and $0.005 \%(\mathrm{w} / \mathrm{w})$ griseofulvin in $70 / 30$ water/acetone $(\mathrm{v} / \mathrm{v})$ (top, middle, bottom, respectively) each deposited onto a blank glass slide. Unlike rotenone, griseofulvin did tend to form crystals on glass slides. 


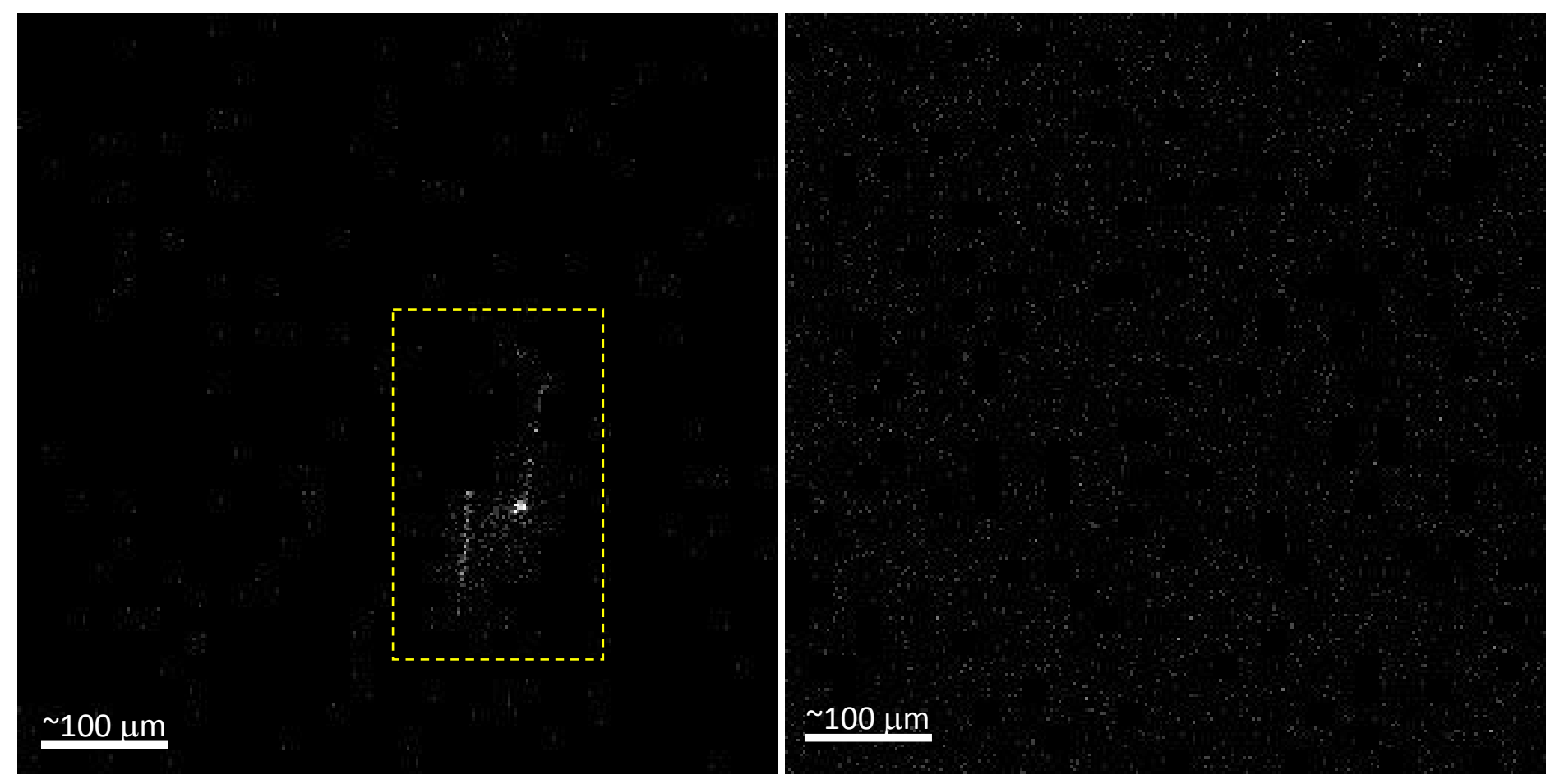

Figure S7: SHG images of wheat grass leaves following the solution-phase deposition of urea. Left: crystals appear for a solution containing only $1.0 \%(\mathrm{w} / \mathrm{w})$ aqueous urea. Right: The same urea solution now also containing $1.5 \%$ $(\mathrm{w} / \mathrm{w})$ polyvinylpyrrolidone (PVP) does not produce crystals following deposition and drying. Images are shown on different brightness and contrast scales.

List of accompanying movies following the crystallization of:

- Griseofulvin on wheatgrass leaf

- Griseofulvin on corn leaf

- Rotenone on soy leaf, trial A

- Rotenone on soy leaf, trial B

- Rotenone on soy leaf, spray-application 